ORIGINAL ARTICLE

\title{
Assessment of sleepiness and sleep debt in adolescent population in Urban Western India
}

\author{
Preeti Devnani MD,D, ABSM *, Neelima Bhalerao MB,BS.,PhD** \\ Sleep Disorders Clinic, Jaslok Hospital Mumbai. \\ Indian J Sleep Med 201 1; 6.4, 140-143
}

\begin{abstract}
Introduction: Adolescence being a crucial and evolving phase of human life is subject to several unique intrinsic and extrinsic stressors contributing to an unstructured and inadequate sleep/wake routine.

Methods: A questionnaire based cross sectional study in 314 school going adolescents aged 13-15 years, grade VIII and IX. Cleveland Adolescent Sleepiness Questionnaire(CASQ) in addition to information regarding hours of sleep obtained were obtained in conjunction with information from school administration.

Results: Twenty five present of adolescents reported falling asleep in class in the morning with $32 \%$ feeling sleepy, $15 \%$ fell asleep on the ride to and from school and $25 \%$ felt sleepy while doing their school assignments at home. Median scores of sleepiness statements, alertness statements and total scores on the CASQ were as follows, 22,13 and 36. Age of the children positively correlated for sleepiness and total scores $(r=0.257, p<0.001)$. No gender differences were noted in these scores or hours of sleep obtained. Sleepiness statement score was higher in children who obtained 4 to 6 hours of sleep (24.8\% of the sample) than the group that obtained 6 to 8 hours of sleep $(59.3 \%$ of the sample) with a p value $<0.05$.

Conclusion: Indian adolescents like their global counterparts also have excessive daytime somnolence and sleep debt which is largely unrecognized. Intervention with education in conjunction with school authorities and families is the need of the hour.
\end{abstract}

Keywords: Sleep debt, adolescents, chronic sleep deprivation

\section{Introduction}

A mongst all the phases of human life, adolescence is perhaps the most significant, signaling transition from childhood to adulthood. This is reflected in the changing lifestyle of adolescents and sleep patterns are far from immune. As demands of shaping

Address for correspondence

Prof. Preeti Devnani MD, D, ABSM

Sleep Disorders Clinic, Department of Neurology and Clinical Neurophysiology, Jaslok Hospital and Research Centre, Mumbai their life and careers grow, proper sleep hygiene is often neglected. There are very few Indian studies which have looked into the sleep patterns of young adults ${ }^{1}$. The consequences of altered sleep wake cycles in adolescents are varied. Apart from excessive sleepiness during school, effects on the cognitive, metabolic and psychosocial aspects are of growing importance, hence the present study was undertaken to create a database of sleep patterns of adolescents in western India, albeit targeting only the urban population. The study was approved by the institutional ethics committee. 


\section{Methods}

Adolescents attending two English medium daytime private schools in Mumbai were included in this study .All students were studying in standard VIII and IX. Participants were acquainted with the study procedure and were administered the Cleveland Adolescent Sleepiness questionnaire (CASQ) during school hours. The confidentiality of the response was maintained.

A total of three hundred and fourteen students completed the CASQ.

\section{Material}

The Cleveland Adolescent Sleepiness questionnaire is an easily administered, self explanatory, self-rating scale of sleepiness patterns. Age and gender of the participants were documented.

\section{Statistical analysis}

Standard statistical tests were applied.

\section{Results}

A total of three hundred and fourteen students participated in the study. Mean age of the participants was 13.8 years (Age range: 13-15). There were 245 boys and 69 girls in this study, with a gender ratio of -

The mean CASQ score was $36.3 \pm 9.75$.

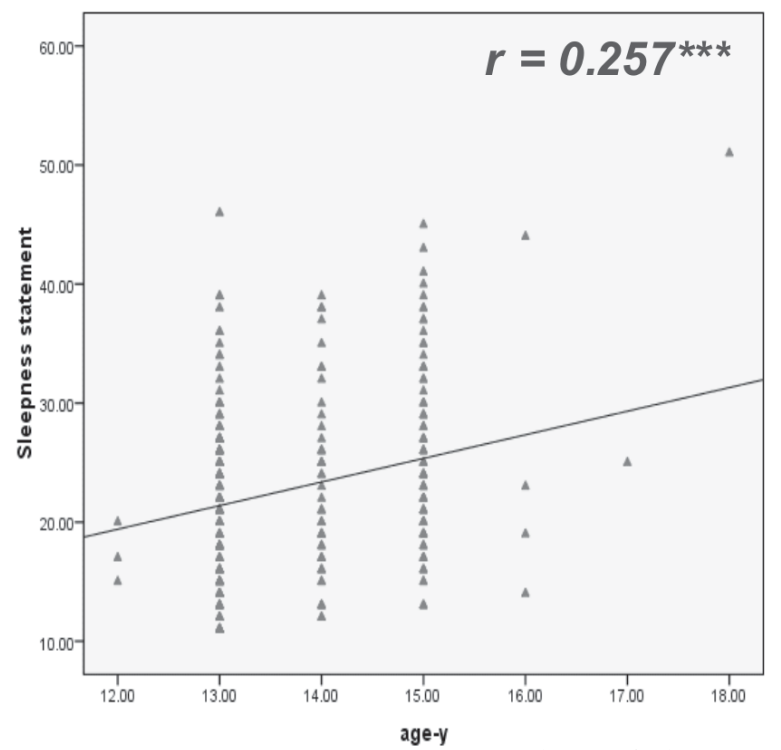

Increasing age was positively correlated with sleepiness as well as total scores $(\mathrm{p}=0.00, \mathrm{r}=0.257)$ however no gender was observed $(\mathrm{p}=0.769)$ in this study. (Fig 1)

\section{Sleepiness statements:}

1. Feel Sleepy during morning hours in school: $32.16 \%$

2. Feel Sleepy in last class: $25 \%$

3. Drowsy in vehicle: $20 \%$

4. Feel Sleepy in the afternoon: $30 \%$

5. Feel Sleepy in evening after school: $55 \%$

6. Feel sleepy on bus ride going for event: $20 \%$

7. Fell asleep in school in morning: $28 \%$

8. Sleepy while doing homework: $25 \%$

9. Asleep in car, bus to and from school: $15 \%$

10.Sleep attacks: $20 \%$ later on understand that they have fallen asleep

11. Sleepy while doing school work: $22 \%$

\section{Alertness statements:}

1. Wide awake whole day: $36.3 \%$

2. Alert during classes: $66 \%$

3. Attend school without feeling tired: 68\%, 30\% feel tired.

4. Wide awake in school: $56.5 \%$

5. Wide awake during last class: $53.8 \%$

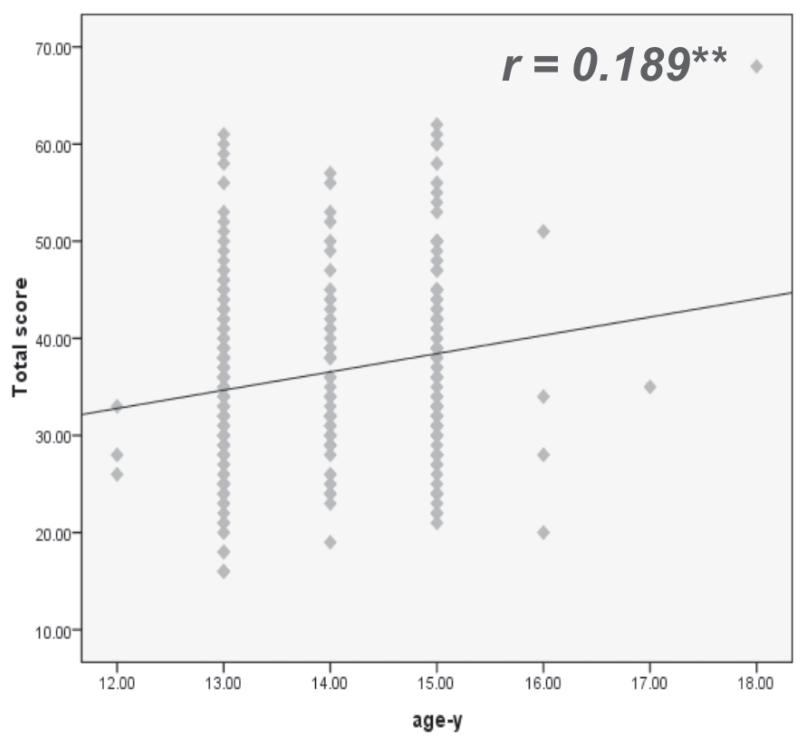

Figure 1: Analysis of Sleepiness and Total Score in relation to Age 
Overall, one third of the study population felt sleepy while attending to school related work, additionally, 20\% almost half of the subjects felt sleepy in the evening.

One third students felt tired. Sleepiness statement score was most significant for children in whom total sleep time was relatively reduced.

$50-60 \%$ of the participants reportedly felt awake and alert in school. Only a third felt awake through the entire day. After school, when stimulating environment was removed, additional $20 \%$ felt sleepy.

Majority i.e. 57\% adolescents slept for 6-8 hours in this study population. Twenty five percent students had significant compromise for sleep duration i.e. slept only for 4-6 hours. Rest of them 15\% had mild shortening of total sleep duration.

Only one child slept less than four hours and six children slept for 10 hours and longer.

However almost all fell below the normal mark required for adolescents age group: 8 hours thirty minutes to 9 hours and 15 minutes.

Significant unrecognized sleep debt was observed.

Gender and the section / class in which they are studying of students (VIII vs IX) did not influence the sleepiness score.

Sleepiness statement score is higher in children who slept for 4 to $6 \mathrm{hrs}$. This was significantly less than those sleeping 6 to $8 \mathrm{hrs}$, whereas the score was similar in children enjoying 8 to 10 hrs of sleep. (Table 1, Table 2)

Total CASQ score was less in those sleeping 6 to $8 \mathrm{hrs}$ as compared to those who sleep for 4 to $6 \mathrm{hrs}(\mathrm{p}<0.05)$. But total score was similar to those who slept for 8 to $10 \mathrm{hrs}$.

\section{Limitations}

This is a preliminary report of an observational study carried out first time in Western India. Though advances in technology are a strong factor in perpetuating sleep disturbances in Indian urban young adults, the techboom has also invaded the rural subjects. An independent study will provide the answer.

This study documents the existence of daytime sleepiness in Indian adolescents, subsequent exploring of the various underlying causative factors is ongoing.

Lifestyle factors may have been probed such as
Table 1: Sleepiness and Alertness statements in correlation with age

\begin{tabular}{|l|l|l|l|l|}
\hline $\mathrm{N}=314$ & age-y & $\begin{array}{l}\text { Sleepiness } \\
\text { stat ement }\end{array}$ & $\begin{array}{l}\text { Alert ness } \\
\text { statement }\end{array}$ & $\begin{array}{l}\text { Total } \\
\text { score }\end{array}$ \\
\hline Mean & 13.8121 & 23.0619 & 13.3344 & 36.2544 \\
\hline Median & 13.0000 & 22.0000 & 13.0000 & 36.0000 \\
\hline $\begin{array}{l}\text { Std. } \\
\text { Deviation }\end{array}$ & .97232 & 7.54364 & 4.00963 & 9.75437 \\
\hline Minimum & 12.00 & 11.00 & 5.00 & 16.00 \\
\hline Maximum & 18.00 & 51.00 & 25.00 & 68.00 \\
\hline
\end{tabular}

Table 2: Summary of sleepiness and Alertness statements in correlation to hours of sleep

\begin{tabular}{|l|l|l|l|}
\hline & $\begin{array}{l}\text { Hou rs of } \\
\text { sleep }\end{array}$ & Mean & $\begin{array}{l}\text { Std. } \\
\text { Deviation }\end{array}$ \\
\hline $\begin{array}{l}\text { Sleepiness } \\
\text { statement }\end{array}$ & 4 to 6 hrs & 25.0563 & 7.86654 \\
\hline & 6 to 8 hrs* & 22.1524 & 7.23878 \\
\hline $\begin{array}{l}\text { Alertness } \\
\text { statement }\end{array}$ & 4 to 6 hrs $10 \mathrm{hrs}$ & 23.1591 & 6.69239 \\
\hline & 6 to 8 hrs & 13.0977 & 4.05550 \\
\hline Total & 4 to $10 \mathrm{hrs}$ & 13.5111 & 3.50728 \\
\hline score & 6 hrs & 39.1343 & 9.91616 \\
\hline & 6 to 8 hrs $*$ & 35.0313 & 9.46588 \\
\hline & 8 hrs & 36.6136 & 8.91880 \\
\hline
\end{tabular}

Independent bedrooms, TV and other gadgets in room, access to internet and mobile phones

Additional documentation of sleep debt may have been brought to light with differentiation in total sleep time on weekends, however with current academic pressure this too is a point of contention and most adolescents are known to burn the candle at both ends with infinite accumulation of chronic sleep debt.

In addition, evaluation of perception of students and their parents about their sleep and health patterns would prove very insightful. 
Worldwide, young adults show a delayed sleep-wake behavior pattern with delayed Sleep Phase Disorder, exacerbated by cultural factors ${ }^{1 .}$ In further identifying causes for shortened Total Sleep Time, circadian pattern must be considered.

Simple, easy to use and validated questionnaires are now available to study adolescent sleep problems ${ }^{2}$.Gupta et al have shown sleep deprivation and symptoms of daytime sleepiness are common in adolescents from North India having linear relation with age ${ }^{3}$. This is in accordance to our study. Similar to our study, gender did not show any influence on sleep parameters in their study.

\section{Conclusion}

Indian adolescents like their global counterparts also have excessive daytime sleepiness and greater sleep debt which is largely unrecognized warranting a bigger study with large sample size.

\section{References}

1. Gradisar M, Gardner G, Dohnt H. Recent worldwide sleep patterns and problems during adolescence: a review and meta-analysis of age, region, and sleep. Sleep Med 2011 Feb; 12(2): 1 10-8.

2. Spilsbury JC, Drotar D, Rosen CL et al. The Cleveland adolescent sleepiness questionnaire: a new measure to assess excessive daytime sleepiness in adolescents. J Clin Sleep Med 2007;3(6):603-12.

3. Seonkyeong Rhie, M.D., Sihyoung Lee, M.D., and Kyu Young Chae, M.D Sleep patterns and school performance of Korean adolescents assessed using a Korean version of the pediatric daytime sleepiness scale Korean J Pediatr. $2011 ; 54(1): 29-35$.
4. Gupta R, Bhatia MS, Chhabra V, Sharma S, Dahiya D, Semalti K, et al. Sleep patterns of urban school-going adolescents. Indian Pediatr. 2008;45: 183-189

5. Waris Qidwai, Sidra Ishaque, Sabeen Shah, and Maheen Rahim Adolescent Lifestyle and Behaviour: A Survey from a Developing Country PLoS One. 2010; 5(9): e 12914

6. Ka-Fai Chung, Miao-Miao Cheung Sleep-Wake Patterns and Sleep Disturbance among Hong Kong Chinese Adolescents SLEEP 2008;31(2):185-194

7. Cheng-Fang Yen, Chih-Hung Ko, Ju-Yu Yen, Chung-Ping Cheng The Multidimensional Correlates Associated With Short Nocturnal Sleep Duration and Subjective Insomnia Among Taiwanese Adolescents SLEEP 2008;31(11):15151525

8. Kotagal S, Pianosi P Sleep disorders in children and adolescents BMJ 2006;332:828-32

9. Raphaële Reine Lydie van Litsenburg, Ruth C. Waumans, Gerrit van den Berg, Reinoud J. B. J. Gemke Sleep habits and sleep disturbances in Dutch children.a population-based study Eur J Pediatr (2010) 169:1009-1015

10. Waleed M Sweileh, lyad A Ali, Ansam F Sawalha 1, Adham S Abu-Taha, Sa'ed H Zyoud, Samah W Al-Jabi Sleep habits and sleep problems among Palestinian students Child and Adolescent Psychiatry and Mental Health 201 1, 5:25

11. Ahmad Ghanizadeh, Mohsen Kianpoor, Mehdi Rezaei, Hadi Rezaei, Rozita Moini, kamran Aghakhani, Jamshid Ahmadi,Seyed Reza Moeini Sleep patterns and habits in high school students in Iran Annals of General Psychiatry 2008, 7:5

12. Xianchen Liu, Zhongtang Zhao, Cunxian Jia, Daniel J. Buysse Sleep patterns and problems among Chinese adolescents Pediatrics 2008;121;1165

13. Edward S Gibson, AC Peter Powles, Lehana Thabane, Susan O'Brien, Danielle Sirriani Molnar, Nik Trajanovic, Robert Ogilvie, Colin Shapiro, Mi Yan,Lisa Chilcott-Tanser "Sleepiness" is serious in adolescence: Two surveys of 3235 Canadian students BMC Public Health2006, 6:1 16 